\title{
Comparing Models of Semantic Search in Concrete and Abstract Categories
}

\author{
David J. Halpern (david.halpern@nyu.edu) \\ Department of Psychology, NYU \\ 6 Washington Place \\ New York, NY 10003 USA \\ Pedro L. Rodríguez (pedro.rodriguez@nyu.edu) \\ Department of Politics, NYU \\ 19 W. 4th St \\ New York, NY 10003 USA
}

\begin{abstract}
Semantic memory retrieval is a function of a search algorithm and a semantic representation. A challenge in studying retrieval has been that different search algorithms on different representations of the same concepts often produce similar results, making theorizing of the underlying processes a slippery endeavor. In this paper we outline a method that bypasses this challenge by estimating representations directly from fluency data. We showcase our method by performing model comparison on existing and new models of memory search. Our results show that the constrained random walk (Austerweil, Abbott, \& Griffiths, 2012) and a random walk without repeats (Millsap \& Meredith, 1987) are able to fit the data almost equally well across several large and diverse datasets.
\end{abstract}

Keywords: semantic memory retrieval; model comparison.

\section{Introduction}

Retrieval of knowledge from memory underlies many cognitive tasks from understanding language to categorization to making evaluative judgments. The semantic fluency task - also known as the continuous response task- is commonly used to study semantic memory retrieval because of the rich data it provides on the nature of the cognitive processes and representations that underlie it. In this task, subjects are given a cue (such as "animals" or "words that start with F") and are required to come up with as many examples of that cue as possible without repeats in a limited amount of time. Through studying this task, cognitive scientists have uncovered many qualitative features of semantic memory retrieval. For instance, we've learned that concepts are likely to be retrieved close in time to other semantically related concepts and that the frequency with which a concept is retrieved is correlated with its frequency in natural language (Troyer, Moscovitch, \& Winocur, 1997). The richness of the data in the semantic fluency task make it particularly well suited for modeling.

Semantic memory retrieval, as captured by the semantic fluency task, has long been understood to be a function of both a semantic representation and a search algorithm for searching through that representation. Much of the literature on semantic memory retrieval revolves around methods to explore one or both of these aspects. A large early literature tested various search algorithms by fitting changes in the rates at which concepts were retrieved over time (Bousfield \&
Sedgewick, 1944). Several of these early papers noted subjects tend to produce items in bursts of semantically related words. To account for this behavioral feature they proposed a two-stage search strategy according to which subjects identified a cluster or "semantic field" (e.g. the "pet" cluster in the "animal" category) and sampled from this cluster until exhausting it. (Bousfield \& Sedgewick, 1944; Gruenewald \& Lockhead, 1980; Graesser \& Mandler, 1978). They would then move on to a different cluster. These models often made vague references to the underlying representations (such as a hierarchical representation) but these were not the direct object of study. A separate set of papers followed the emergence of multidimensional scaling tools. These largely ignored the search process and used multidimensional scaling tools to estimate the semantic representations from fluency lists (Chan et al., 1993; Rubin \& Olson, 1980). Estimated representations were validated by comparing them to human performance in various other behavioral tasks. This early literature then largely focused on either the search algorithm or the semantic representation, limiting their ability to make predictions from behavioral results.

The advent of increased computing power and easy access to large datasets, including text corpora and word association data, made it possible to explore both search and representation. Today semantic memory retrieval is most commonly studied using a two-step process: (1) estimate a representation, customarily a network or a vector space, using either a corpus or free-word association data. (2) Simulate a search process on this representation. The search algorithm is then evaluated by comparing its output to behavioral data, often semantic fluency lists. Building on the earlier findings suggesting that memory was organized in clusters (Bousfield \& Sedgewick, 1944) and search processes switch from global to local cues (Gronlund \& Shiffrin, 1986), Hills, Jones, and Todd (2012) propose a dynamic local-to-global search strategy similar to how animals forage for food in space. To evaluate this new search algorithm they first estimate two separate representations, one derived straight from a hand coded categorization scheme for the category "animals" (Troyer et al., 1997) and another derived using a co-occurrence model (BEAGLE) on a Wikipedia corpus. They found the combination of their proposed search algorithm with the representations employed could reproduce many of the features ob- 
served in semantic fluency data, including the relationship between item similarity and response time as well as the likelihood of switching clusters.

Austerweil et al. (2012) followed the same two-step strategy but with a different representation (a semantic network constructed from word association norms) and a different search algorithm (a simple random walk). They found that they could replicate the same behavioral features as in Hills et al. (2012). This result served to highlight a fundamental challenge of modeling semantic memory retrieval: "different algorithms operating over different representations can produce the same predictions" (Austerweil et al., 2012; Anderson, 1978). Thus, attempts to model search by assuming a particular representation could be biasing the results.

Millsap and Meredith (1987), although preceding many of these papers, had already offered a potential solution to the challenge highlighted above. Instead of assuming a representation and then evaluating different search algorithms relative to the semantic fluency data, they proposed estimating the representation directly from the fluency data assuming a given search strategy. However, because of computational constraints, they use a biased estimation procedure and do not compare their model to any others (presumably because few others had been proposed). In a much more recent paper Jun, Zhu, Rogers, and Yang (2015) assume a censored random walk strategy and propose an unbiased estimator to compute the representation directly from the fluency lists. However, they only compare it's performance to two other biased procedures for estimating semantic representations. Zemla, Kenett, Jun, and Austerweil (2016) proposed extending the model of Jun et al. (2015) to account for the representations of individual subjects but here the goal was to investigate the representations rather than the search process and no models of search were compared.

Building on these advances in this paper we outline a method for comparing models of semantic memory search that attends to the shortcomings of existing methods. Our method can be summarized as follows, given alternative search algorithms: (1) divide the set of fluency lists into a training and a test set. (2) Estimate model parameters directly from the fluency data for each of the proposed algorithms. The estimated parameters under a given algorithm constitute the semantic representation for that algorithm. (3) Evaluate model performance on the test set and identify the search algorithm that best predicts the held-out set. This procedure guarantees that all algorithms are compared using the same data and with respectively optimized parameters. Unlike the two-stage method outlined above, this procedure does not require a separate corpus, which may not accurately capture the semantic space involved in fluency tasks, or word-association data which can be expensive and time-consuming to acquire. This makes this method much more versatile, allowing us to explore semantic representations of specific concepts, in distinct contexts, for particular sub-populations and as a function of potential treatment assignments (e.g. a prime).
We use this procedure to directly compare alternative search algorithms, some which have been proposed in the literature as well as new ones. We make two additional contributions to the memory retrieval literature. First we extend our model comparison to abstract concepts (e.g. welfare, American values, government). In previous papers on semantic fluency, data have focused almost exclusively on a small set of concrete categories such as "animals", "foods", "US states" or some subset of those (e.g. "fruits", "four-legged animals"). This made sense since it was easier for single subjects to give long lists on these categories and the data were easier to interpret (for instance, it was easier to find clusters). However, it is possible that semantic organization and retrieval differ in different parts of semantic space. In particular, abstract categories may use different search processes than the concrete categories used in most studies. Second, we also perform model comparison on different groups of people as defined by political party identification and gender. A growing body of literature has identified individual differences in semantic representations. It is possible that these differences extend to or indeed are drive by differences in search algorithm.

Our results confirm the censored random walk to perform the best or tied with the best in terms of predicting the log-likelihood of held-out lists in model comparisons on all datasets. However, we also find a much simpler model, a random walk in which the search returns to the cue word upon landing on an already emitted word, to perform statistically on par with censored random walk while greatly reducing computational costs.

\section{Measurement models}

In this paper we focus on network representations of semantic memory. This is not because we want to take an ideological stance but because the current existing models for inferring semantic representations involve networks. It should be possible to alter these models to estimate something more like a semantic space (e.g. BEAGLE (Jones \& Mewhort, 2007) by putting constraints on the network transition matrix. We leave developing those models to future work.

In general, to estimate a semantic network, we want to estimate a transition matrix $\mathbf{P}$ where each element of the matrix $P_{i j}$ represents the probability of transitioning from word $i$ to word $j$ in one step on the network. Given $\mathbf{P}$, we can compute the likelihood of each list in our dataset and use maximum likelihood or Bayesian inference to infer the parameters of our semantic network. However, for many models of search, the likelihood of emitting a semantic fluency list is not simple to compute because after the second word in the list, the probability of transitioning between two words $i$ and $j$ is not just $P_{i j}$. This is because in the semantic fluency task, participants are not allowed to repeat a word so the probability of the next word depends on the words the have already been said. Indeed, in any generative model of the task, the likelihood of emitting a word that has been said previously should be 0 although various models deal with how to treat these words that 
have already been said differently. Below we describe several search processes that have been suggested in the literature.

\section{CRW}

This search process was first described in Austerweil et al. (2012) where it was defined as a random walk on the network but where words are only added to the semantic fluency list $a$ on the first time the search process reaches them. We follow Zemla and Austerweil (n.d.) in calling this a censored random walk (CRW). This means at any point, if the search process is currently on node $i$, it takes a step to a new node $j$ with probability $P_{i j}$. If $j$ corresponds to a word that is not in $a$, that word is entered into the list. However, if $j$ is in $a$, no word is added to the list. In both cases, the search process continues to another node $k$ with probability $P_{i k}$. This makes computing the likelihood very difficult because in a network where every word is connected to each other (with different strengths) there might be infinite possible paths through nodes already in $a$ before arriving at a new word that is not yet in $a$. Jun et al. (2015) were the first to come up a method to integrate over these paths and compute a likelihood which they called INVITE. Given a transition matrix $\mathbf{P}$, we can compute the probability of the $(k+1)$ th element in a semantic fluency list $a, \operatorname{Pr}\left(a_{k} \mid a_{1: k}, \mathbf{P}\right)$, by first rearranging the columns of $\mathbf{P}$. Let $\mathbf{Q}^{(\mathbf{k})}$ be the rows and columns of $\mathbf{P}$ corresponding to transitions between words that have already occurred in the list up to word $k$, i.e. $\mathbf{Q}^{(\mathbf{k})}=\mathbf{P}\left[a_{1}: a_{k}, a_{1}: a_{k}\right]$. Let $\mathbf{R}^{(\mathbf{k})}$ be the rows and columns corresponding to transitions from words that have already occurred to words that have not, i.e. $\mathbf{R}^{(\mathbf{k})}=\mathbf{P}\left[a_{1}: a_{k}, a_{k+1}: a_{M}\right]$. Now define a new matrix:

$$
B^{(k)}=\left(\mathbf{I}-\mathbf{Q}^{(\mathbf{k})}\right)^{-1} \mathbf{R}^{(\mathbf{k})}
$$

where I is the identity matrix of size $k$. Jun et al. (2015) show that the probability of starting from word $a_{i}$ for $i \in(1 \ldots k)$ and emitting a word $a_{j}$ not already in the list (i.e. $j \in(k+1 \ldots M)$, integrating over all possible paths, is equal to $B_{i j}^{(k)}$. If the $k$ th column of $\mathbf{Q}^{(\mathbf{k})}$ corresponds to $a_{k}$ and the first column of $\mathbf{R}^{(\mathbf{k})}$ corresponds to the word $a_{k+1}$ then we can compute

$$
\operatorname{Pr}\left(a_{k+1} \mid a_{1: k}, \mathbf{P}\right)=B_{k 1}^{(k)} .
$$

We can now compute the probability of a whole list by first selecting the first word according to a distribution $\pi$ and then computing the probabilities of the rest of the list using the above method.

\section{CRW-LG}

Several papers (Hills et al., 2012; Gronlund \& Shiffrin, 1986), claim that semantic memory retrieval depends on the interplay between two separate processes: one that retrieves based on similarity to a global cue and one that retrieves based on similarity a local cue. Similarity to the local cue depends on the previous word mentioned in the list while similarity to the global cue only depends on general properties of the word. In Hills et al. (2012), using the global cue was modeled as choosing the next word with probability proportional to general frequency and using the local cue was modeled as corresponding to choosing the next word with probability proportional to similarity to the previous word on the list. We here derive a measurement model of a local-to-global switching process, which we call CRW-LG (for censored random walk-local to global). In a local-to-global model, we can assume that choosing the first word on the list necessarily only depends on the global cue. Therefore, we assume that using the global cue is the same as using the probabilities associated with selecting the first word. In the CRW model estimated in Jun et al. (2015), this is the vector $\pi$. However, to make estimation easier we make a small change to the CRW model and treat the cue word (e.g. "animals") as a node in the network. This means that the cue word is included in the transition matrix. This is also a slight conceptual change from the above INVITE model in that any transition integrates over the probability of stepping through the node for "animals." In this formulation, using the global cue is the same as jumping to the cue word and proceeding through the network from there. Inspired by the implementation of a jumping model from Austerweil et al. (2012), we assume that there is a probability $\rho$ of using the global cue. Our likelihood is then the same as equation 3 from Austerweil et al. (2012), i.e. if the cue word is "animals",

$$
\begin{aligned}
& \operatorname{Pr}\left(a_{k+1} \mid C=\right.\text { "animals", }\left.a_{k}=a_{k}, \mathbf{P}\right)= \\
& \rho \operatorname{Pr}\left(a_{k+1} \mid a_{k}=\text { "animals"P }\right)+ \\
&(1-\rho) \operatorname{Pr}\left(a_{k}+1 \mid a_{k}=a_{k}, \mathbf{P}\right)
\end{aligned}
$$

Using the INVITE, we can fill this in as follows:

$$
\begin{aligned}
\operatorname{Pr}\left(a_{k+1} \mid C=\text { "animals", } a_{k}=a_{k}, \mathbf{P}\right)= \\
\qquad \rho B_{i 1}^{(k)}+(1-\rho) B_{k 1}^{(k)}
\end{aligned}
$$

where $i$ is the column of $\mathbf{Q}^{(\mathbf{k})}$ corresponding to the cue word and $k$ is the column corresponding to the previous word.

In Hills et al. (2012), the switches between local to global are thought to be strategic and depend on the availability of relevant words in the current cluster of semantic space. Thus, Abbott, Austerweil, and Griffiths (2015) are careful to emphasize that this version of a jumping model when simulated on an existing semantic network is not the same as that used in Hills et al. (2012) because the decisions to jump are random. However, we claim that from the perspective of a measurement model where we simultaneously estimate $\mathbf{P}$ and $\rho$, we are agnostic as to the reasons for jumping. If the strategic local to global switching model is correct, as long as the probability of making a strategic jump is relatively constant throughout the list, this measurement model should be able to recover the parameters and perform better than the $C R W$ model described above. However, it is unlikely to be the case that strategic jumps are as common early in the list as later in the list and we describe one attempt to improve on this model below. 


\section{CRW-LG-strategic}

This model is the same as INVITE-LG but with strategic jumps. Hills et al. (2012) make an analogy between semantic search and foraging decisions. In foraging for food, for instance, if there are few berries left in a patch it makes sense to begin looking for a different patch. Similarly, if there are very few unsaid words left are similar to the local cue (i.e. near the previous word in semantic space), it makes sense to use the global cue instead. However, in the Hills et al. (2012) study, they first restricted the semantic space to words that satisfy the cue word (e.g. "animals"). We argue that this definition of strategic switching only makes sense in this limited setting. Otherwise, following a chain of similarity through a highlyconnected semantic network, it would be easy to move to a word that is inappropriate for the cued concept (e.g. "horse", "cow", "farm"). Another sense in which switches might be strategic is that they switch to the global cue when the search process has strayed too far from the cue word. In order to test whether people are strategic in this sense, we need a measure of how far the current word is from the cue word. As a proxy, we use the probability of switching to the current word from the cue and set $\rho$ in the above INVITE-LG model to $1-P_{1 k}$.

\section{RW-without-repetition}

This model, a random walk (RW) without repeats, was first described described in Millsap and Meredith (1987). Essentially, the search process follows the transition probabilities in $\mathbf{P}$ but respects the constraint that there are no repeats. This can be thought of as a strategy that takes a random walk on the network but if it encounters a node corresponding to a word that has already been said in the list, it backtracks to the previous node. In contrast, the CRW continues through the network. The probability of transitioning from word $a_{k}$ to $a_{k+1}$ under this model is simply

$$
\operatorname{Pr}\left(a_{k+1} \mid a_{1: k}, \mathbf{P}\right)=\frac{P_{k(k+1)}}{\sum_{i=k+1}^{M} P_{k i}}, k<M
$$

\section{BoW-without-repetition}

This model assumes that there is a fixed probability distribution for retrieving words associated with a cue (a Bag of Words model). This is no longer a "network" per se but could be an algorithm operating on a network if, after emitting every word, the searcher always returns to the cue node. A model like this was proposed in ? (?) under the name Node Degree Search where the probability of retrieving a word was proportional to it's long run probability of retrieval in the network. However, we again alter this model so that it can generate possible lists by enforcing the without repetition constraint. Thus the probability of transitioning from word $a_{k}$ to $a_{k+1}$ is

$$
\operatorname{Pr}\left(a_{k+1} \mid a_{1: k}, P\right)=\frac{P(k+1)}{\sum_{i=k+1}^{M} P_{i}}, k<M
$$

\section{Estimation}

For each of these models, on all of our datasets, we perform maximum likelihood estimation using LBFGS in rStan. Each model has roughly the same number of parameters, namely an $n$-by- $n$ matrix $\mathbf{P}$ (where $n$ is the total number of words used in the dataset) corresponding to the transition matrix. In the case of CRW-LG, we estimate an additional $\rho$ parameter. For BoW-without-repetition we only need to estimate a vector $P$ of length $n$. It is also easier to estimate parameters as unconstrained real numbers and then transform them into probabilities so rather than estimating the exact parameters of $\mathbf{P}$, we estimate parameters $\beta$ and then row normalize to turn them into $\mathbf{P}$. For models with so many parameters using unregularized estimates is unlikely to perform well, especially if the data are small. Even if we have several hundred lists, there may be words or transitions between words that are observed only a few times. Therefore, we follow Jun et al. (2015) in using penalized maximum likelihood. We find it easier to think about penalties as priors - equivalent to the penalty used in Jun et al. (2015), we place a Gaussian prior on the $\beta$ parameters with mean 0 and variance $2 / C$ with $C$ as a hyperparameter. For the $\pi$ parameters in the CRW models, Jun et al. (2015) use the raw maximum likelihood estimate rather than using the penalty (equivalent to using a uniform Dirichlet prior). However, we find, especially with smaller datasets, the models fit better when we use the Gaussian prior for these parameters as well. For the, CRW-LG-strategic model, we also place a $\operatorname{Beta}(5,1)$ prior on $\rho$ with the assumption that global transitions are relatively rare. Finally, Millsap and Meredith (1987) rather than estimation using maximum likelihood, propose using less optimal but computationally less expensive methods for estimating the network that relies only on the counts of occurrences. These methods involve either counting the total empirical transitions between each word, which results in a biased estimate due to the constraint on repetitions, or counting the empirical transitions between just the first and second word, which is unbiased but ignores a lot of data and so is a more variable estimate. In Jun et al. (2015) these models are labeled RW for Random Walk and FE First Emission. We can compare these methods of estimation by still computing the likelihoods of heldout lists under the estimated semantic representation. We also estimate BoW, a bag of words model that does not respect the repetition constraint.

\section{Data}

We compare the models on seven datasets. Two were collected as part of the Wisconsin Longitudinal Survey, a survey of over 10,000 participants who graduated from high school in Wisconsin in 1957. The most recent two rounds of the survey collected semantic fluency data for the categories "foods" and "animals" from 4624 and 4714 subjects respectively. The other five were collected by the authors as part of a study on representations of political concepts. The specific concepts tested were "American values," "government," "welfare," "Democrat" and "Republican." The data were collected on mTurk from 1056 subjects who performed semantic fluency tasks for all five topics. 


\section{Results}

To do the model comparison on the Wisconsin data, we followed Jun etal.(2015) of holding out $10 \%$ of our data as a test set. Using the rest of the data as a training set, we performed 5 -fold cross validation to recover the penalty hyperparameter $C$ with that gave the highest held out log likelihood for each model. Finally, we estimated each model using the best fitting $C$ parameter on the entire training set. We used this model to compute the log likelihood of every list in the test set under each model. Our results are shown for both categories in the WLS data in Figure $1 .{ }^{1}$ On this dataset, CRW is numerically the best fitting model and with a dataset this large, using a Gaussian prior did not make a difference. However, nearly all CRW models and RW without repetition models fit nearly as well and are not significantly different from each other. The CRW-LG model performed very similarly to CRW because the estimate for the $\rho$ parameter when training on the entire training set was .0000014 in the foods category and .021 in the animals category so differences between that model and CRW were very subtle. However, relative to CRW, RW without repetition has the added advantage of a much lower computational cost - in our fitting it was on average 10 times faster to fit ( 14 minutes vs. 13 hours for CRW) likely because computing it's likelihood did not involve large matrix inversion. Secondly, CRW-LG-strategy does very poorly. It is possible that this is due to the lack of flexibility in the relationship between jumping probability and the transition matrix. Future work should investigate whether this can be remedied with a parameter that scales this relationship.

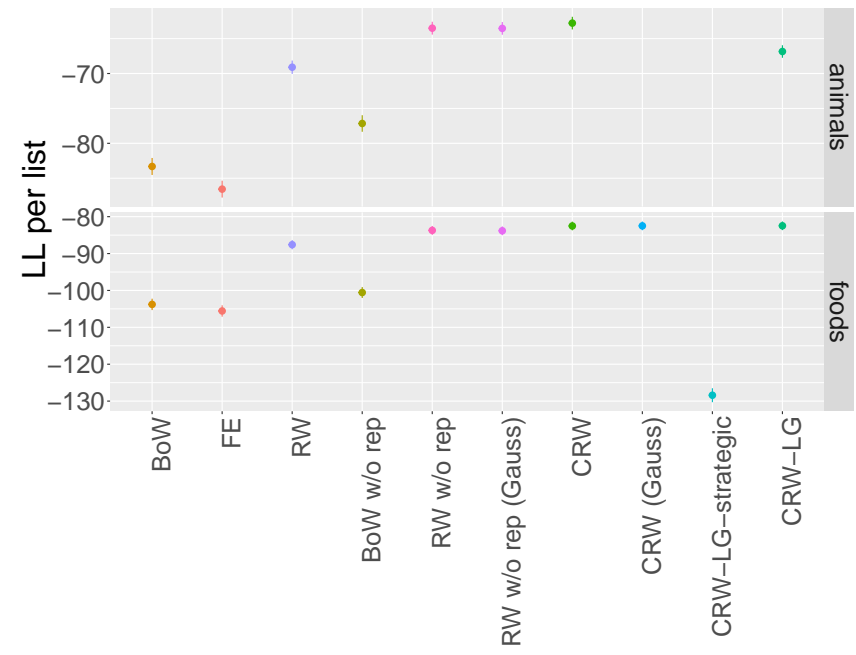

Figure 1: Heldout log likelihood for each model in both Wisconsin datasets

For the data on political concepts, because the data was relatively smaller, we simply fixed $C$ at 1 so as to not lose data

\footnotetext{
${ }^{1}$ Unfortunately, CRW (Gauss) and CRW-LG-strategic crashed while estimating the animals model and since the models require a couple of days to converge, could not be included at the time of submission.
}

in the validation procedure. This value led to a fair amount of regularization but this was appropriate because the data for some transitions was relatively sparse. For each concept, we divided up our data into ten equally sized folds. We fit each model on nine of the folds and evaluated the log likelihood on the tenth. We repeated this process ten times, once for each fold. We here plot the results by topic and averaged over party in Figure 2 as well as by party and averaged over topic Figure 3. We can see that qualitatively, across all divisions of the data, the same models fit well, namely the CRW and RW without repetition. CRW-LG performed as well as CRW because the median parameter estimate for $\rho$ across all folds was .000016 so they were effectively the same model.

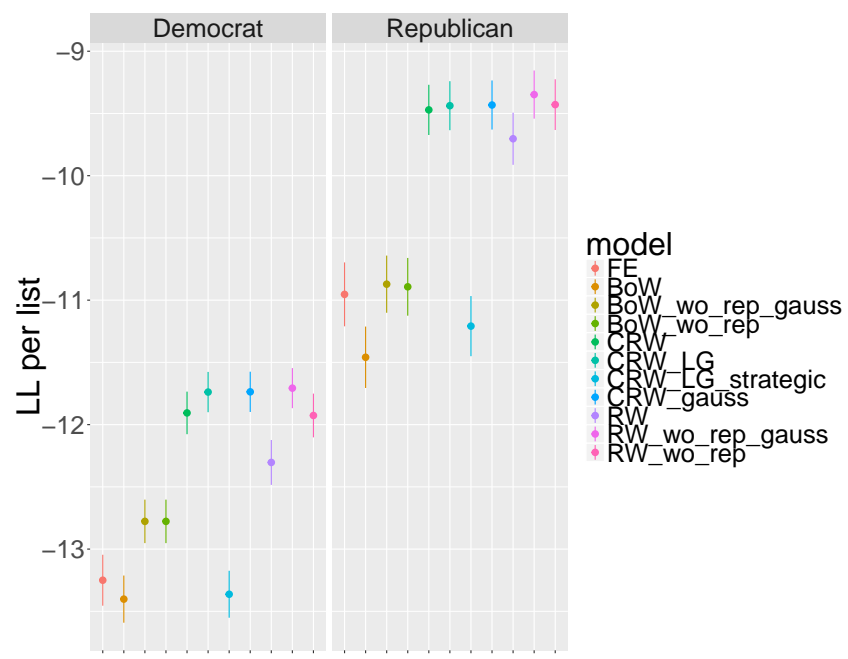

Figure 2: Heldout log likelihood for each model across both parties averaging over topic

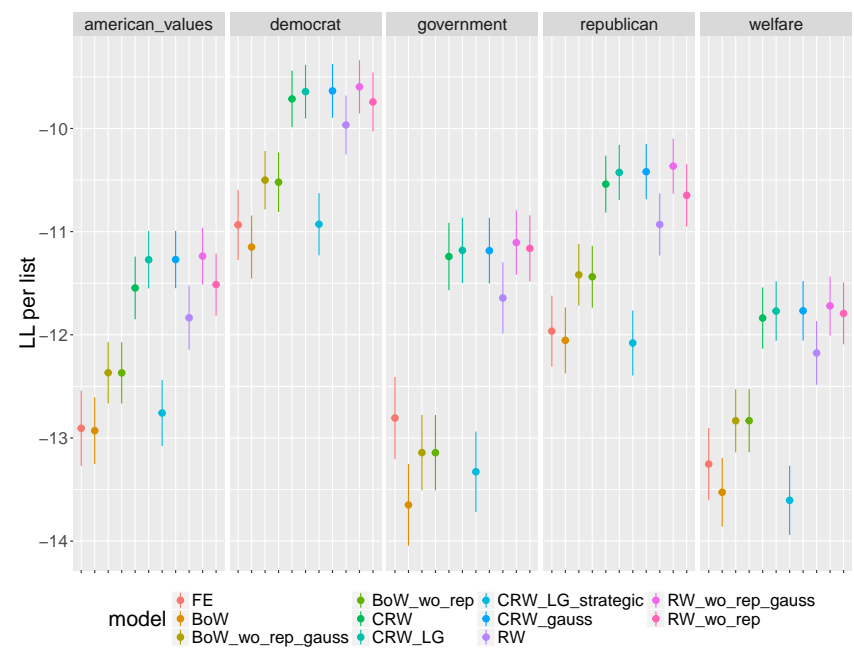

Figure 3: Heldout log likelihood for each model across all topics 


\section{Discussion}

In this paper, we have proposed measurement models of semantic memory retrieval as a method for evaluating search strategies and derived several new measurement models. We demonstrate that several families of models in the literature, namely the constrained random walk (?, ?) and a random walk without repeats (Millsap \& Meredith, 1987) are able to fit the data almost equally well across several large and diverse datasets. However, the random walk model is much less computationally costly making it an attractive model for researchers who may be simply interested in estimating a semantic network from data rather than comparing search algorithms. While we had hoped to be able to compare the major competing models, some implementational issues may have prevented us from making any strong statements. CRW-LG rarely had a high enough $\rho$ parameter to distinguish its predictions significantly from the CRW model. It is possible that our prior was too strong or that we did not have enough data to distinguish the models. Future work should examine the sensitivity of the estimates to the model specification. CRWLG-strategic may have been too misspecified and was not flexible enough to predict the data. However, we hope that this work inspires further work on developing and comparing these type of models. In addition, we by no means think this is the only way to model and learn about semantic search strategies. It would be valuable in future work to compare our results with those found in Zemla and Austerweil (n.d.) and Nematzadeh, Miscevic, and Stevenson (2016) comparing search algorithms on pre-specified networks that were developed either from word associations or learned from child speech data. These have other desirable properties such as a learning algorithm for constructing the network representation that is used to compare models. While our focus here was on comparing search strategies, the inferred representation from these models could also be of interest. In particular, it may be useful to compare the representations to those derived from corpora (i.e. Jones and Mewhort (2007)) to understand the differences.

\section{Acknowledgments}

This research was generously supported by the George Downs Prize grants as well as an NSF grant BCS-1255538 and a John S. McDonnell Foundation Scholar Award to Todd M. Gureckis.

\section{References}

Abbott, J. T., Austerweil, J. L., \& Griffiths, T. L. (2015). Random walks on semantic networks can resemble optimal foraging.

Anderson, J. R. (1978). Arguments concerning representations for mental imagery. Psychological review, 85(4), 249.

Austerweil, J. L., Abbott, J. T., \& Griffiths, T. L. (2012). Human memory search as a random walk in a semantic net- work. In Advances in neural information processing systems (pp. 3041-3049).

Bousfield, W. A., \& Sedgewick, C. H. W. (1944). An analysis of sequences of restricted associative responses. The Journal of General Psychology, 30(2), 149-165.

Chan, A. S., Butters, N., Paulsen, J. S., Salmon, D. P., Swenson, M. R., \& Maloney, L. T. (1993). An assessment of the semantic network in patients with alzheimer's disease. Journal of Cognitive Neuroscience, 5(2), 254-261.

Graesser, A., \& Mandler, G. (1978). Limited processing capacity constrains the storage of unrelated sets of words and retrieval from natural categories. Journal of Experimental Psychology: Human Learning and Memory, 4(1), 86.

Gronlund, S. D., \& Shiffrin, R. M. (1986). Retrieval strategies in recall of natural categories and categorized lists. Journal of Experimental Psychology: Learning, Memory, and Cognition, 12(4), 550.

Gruenewald, P. J., \& Lockhead, G. R. (1980). The free recall of category examples. Journal of Experimental Psychology: Human Learning and Memory, 6(3), 225.

Hills, T. T., Jones, M. N., \& Todd, P. M. (2012). Optimal foraging in semantic memory. Psychological review, 119(2), 431.

Jones, M. N., \& Mewhort, D. J. (2007). Representing word meaning and order information in a composite holographic lexicon. Psychological review, 114(1), 1.

Jun, K.-S., Zhu, X., Rogers, T. T., \& Yang, Z. (2015). Human memory search as initial-visit emitting random walk. In Advances in neural information processing systems (pp. 1072-1080).

Millsap, R. E., \& Meredith, W. (1987). Structure in semantic memory: A probabilistic approach using a continuous response task. Psychometrika, 52(1), 19-41.

Nematzadeh, A., Miscevic, F., \& Stevenson, S. (2016). Simple search algorithms on semantic networks learned from language use. arXiv preprint arXiv:1602.03265.

Rubin, D. C., \& Olson, M. J. (1980). Recall of semantic domains. Memory \& Cognition, 8(4), 354-366.

Troyer, A. K., Moscovitch, M., \& Winocur, G. (1997). Clustering and switching as two components of verbal fluency: evidence from younger and older healthy adults. neuropsychology, 11(1), 138.

Zemla, J. C., \& Austerweil, J. L. (n.d.). Modeling semantic fluency data as search on a semantic network.

Zemla, J. C., Kenett, Y. N., Jun, K.-S., \& Austerweil, J. L. (2016). U-invite: Estimating individual semantic networks from fluency data. In Proceedings of the 38th annual meeting of the cognitive science society (pp. 1907-1912). 